\title{
GREEN'S FUNCTIONS FOR DISSIMILAR OR HOMOGENEOUS MATERIALS CONTAINING INTERFACIAL CRACK, UNDER AXISYMMETRIC SINGULAR LOADING SOURCES
}

\author{
D.G. PAVLOU \\ Department of Mechanical and Structural Engineering and Materials Science, University of Stavanger, Norway.
}

\begin{abstract}
A review of Green's functions for dissimilar or homogeneous elastic space containing penny-shaped or annular interfacial cracks under singular ring-shaped loading sources is presented. The solutions are based on fictitious singular loading sources and superposition of the fundamental solutions of the following two problems: (a) Dissimilar elastic solid without crack under singular source, and (b) Dissimilar elastic solid containing crack under surface tractions. The above Green's functions have the following advantages: (i) No multi-region BE modeling for the dissimilar material is necessary, and (ii) No discretization of the crack surface is necessary. Numerical examples are presented and discussed. Keywords: axisymmetric loading, crack, dissimilar material, green's functions.
\end{abstract}

\section{INTRODUCTION}

The usual methodologies for numerical solution of crack problems are based on high-density mesh. For analyzing interfacial cracks in dissimilar materials, apart from high-density mesh, multi-domain modeling is necessary [1,2]. The multi-domain modeling is mostly known as a technique for solving non-homogeneous material problems. Gao et al. [2] have proposed a three-step solution technique where normalized displacements and tractions are formulated for each sub-domain. Their procedure is based on the following steps: (a) they eliminate internal variables at the individual domain level, (b) they eliminate boundary unknowns defined over nodes used only by the domain itself, and (c) they establish the system of equations according to the compatibility of displacements and equilibrium of tractions at common interface nodes. Ribeiro and Paiva [3] have implemented an alternative multi-region technique. Establishing relations between the displacement fundamental solutions of the different subdomains, they analyze the sub-domains as one unique solid, not requiring equilibrium or compatibility equations. Ramšak and Škerget $[4,5]$ have proposed an efficient 3D multidomain modeling for solving the Laplace and the Poisson equation. They discretized the integral boundary equation using mixed boundary elements. The multi-domain technique has also been used for BE modeling of heat transfer [6] and fluid mechanics [7] problems. The multi-domain methodology seems to be an effective method for bi-materials containing interfacial cracks as well $[8,9]$. Phan and Mukherjee [10] have implemented the boundary contour method (BCM), which offers a further reduction of the computational cost. Zhao et al. [11] have extended the displacement discontinuity method to analyze interfacial cracks in magneto-electro-elastic bi-materials. Since non-homogeneous materials (e.g. bi-materials or composites) have currently many engineering applications, the analysis of interfacial cracks $[12,13]$ is always an interesting subject for research. In the mechanical engineering practice, axisymmetric structural parts (e.g. shafts) containing internal or external interfacial cracks are often analyzed. For these specific cases of non-homogeneous axisymmetric solids containing axisymmetric crack under axisymmetric loading, the use of Green's functions [14] has 
important benefits: (a) reduction of the dimensionality of the problem from 3D to $1 \mathrm{D}$, (b) substantial reduction of the number of nodes because no multi-domain modeling is necessary, and (c) substantial improvement of the accuracy and reduction of the computing cost because no discretization of the crack surface is necessary. However, the price for the above benefits is the complexity of the mathematical derivation of the Green's functions. A review of methods for Green's functions derivation for axisymmetric dissimilar or homogeneous materials containing cracks under ring-shaped singular loading sources, developed by the author, is the aim of the present work.

\section{LOADING SOURCES}

Cracked axisymmetric solids subjected to torsional and axial loads (e.g. shafts) can be analyzed with the aid of Green's functions. Suitable singular loading source for deriving the field of displacements in an infinite medium in order to create the BE model of finite axisymmetric solids is the ring-shaped one containing torsional, axial, and radial components (Fig. 1). It is more convenient to derive the Green's functions for the three components of the loading source separately and then to superpose the solutions. For the torsional (tangential) loading source $q_{9}$, complete Green's functions exist for the case of dissimilar materials containing internal penny-shaped or external annular interfacial crack (Fig. 2a and b). For the axial and radial sources $\mathrm{q}_{\mathrm{z}}, \mathrm{q}_{\mathrm{r}}$ respectively, only the analytic solution of the stresses $\sigma_{\mathrm{zz}}$ is available for a homogeneous medium containing internal penny-shaped or external annular crack (Fig. 3). However, for the purpose of finding the mode I stress intensity factor $\mathrm{K}_{\mathrm{I}}$, this stress component is sufficient [15].

\section{A REVIEW OF GREEN'S FUNCTIONS}

Analytic solutions for the problems shown in Figs 2a,b and 3a,b have been derived by the author in refs [16-19]. The procedure for the derivation of the above Green's functions is

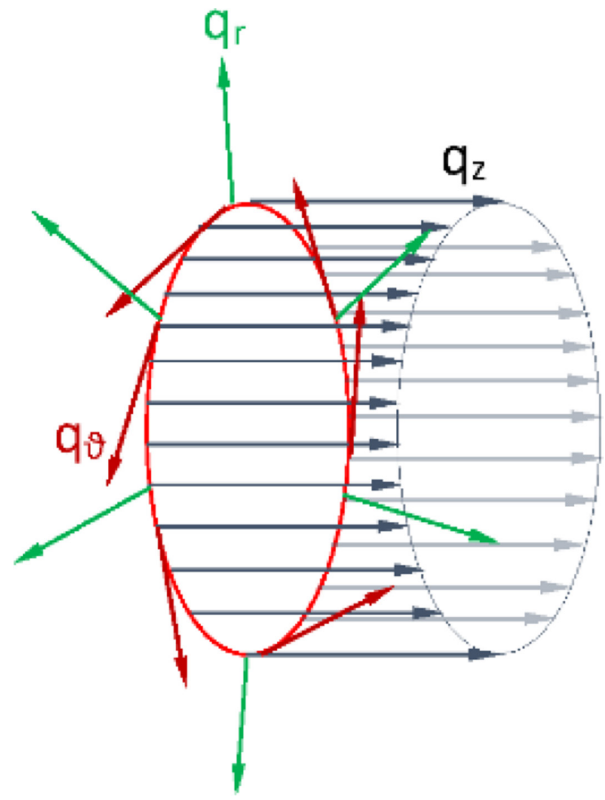

Figure 1: Ring-shaped singular loading source. 


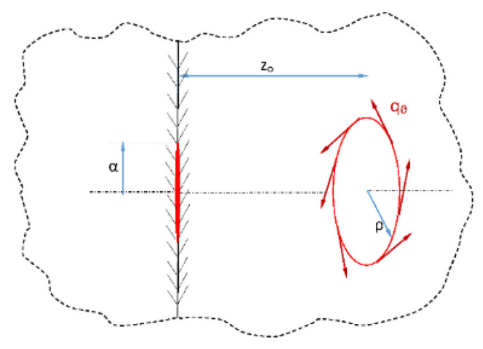

(a)

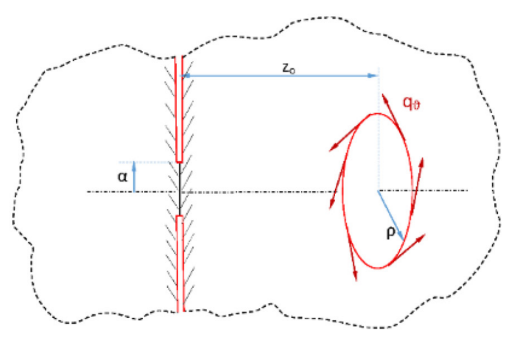

(b)

Figure 2: Dissimilar material under singular tangential ring-shaped source containing: (a) internal penny-shaped interfacial crack, (b) external annular interfacial crack.

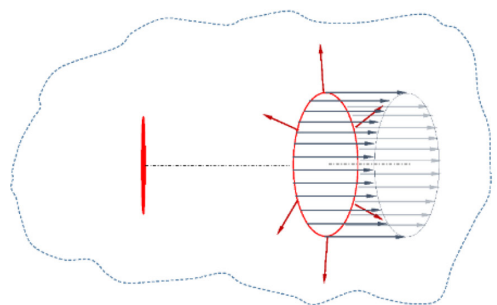

(a)

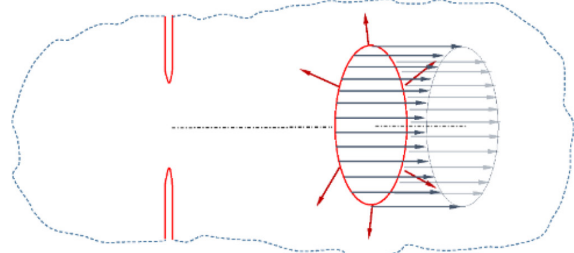

(b)

Figure 3: Homogeneous material under axial and radial loading sources containing: (a) internal penny-shaped crack, (b) external annular crack.

based on the superposition of two partial solutions: (a) the solution of the medium without crack under the ring-shaped singular source, and (b) the solution of a cracked medium without ring-shaped source, where the crack surfaces are subjected to opposite tractions as derived from the first problem at the prospective crack site (Fig. 4).

For the case of dissimilar material containing interfacial crack under tangential ring-shaped source, a special technique for the solution of the first problem based on fictitious loading sources has been used [16, 17].

\subsection{Solution for the un-cracked dissimilar elastic medium}

According to this technique, the dissimilar medium is decomposed to two half spaces. One half space is loaded by the actual ring-shaped source as well as the surface stresses due to the contact of the other half space. The second half space is loaded only by the surface contact stresses (Fig. 5). Since analytic solution for a homogeneous medium subjected to ring-shaped tangential source is available [20], any half space can be considered as a sub-domain of the homogeneous medium subjected to two equal and symmetrically located (with respect to the prospective free surface of the half space) loading sources (Fig. 6). Therefore, the 

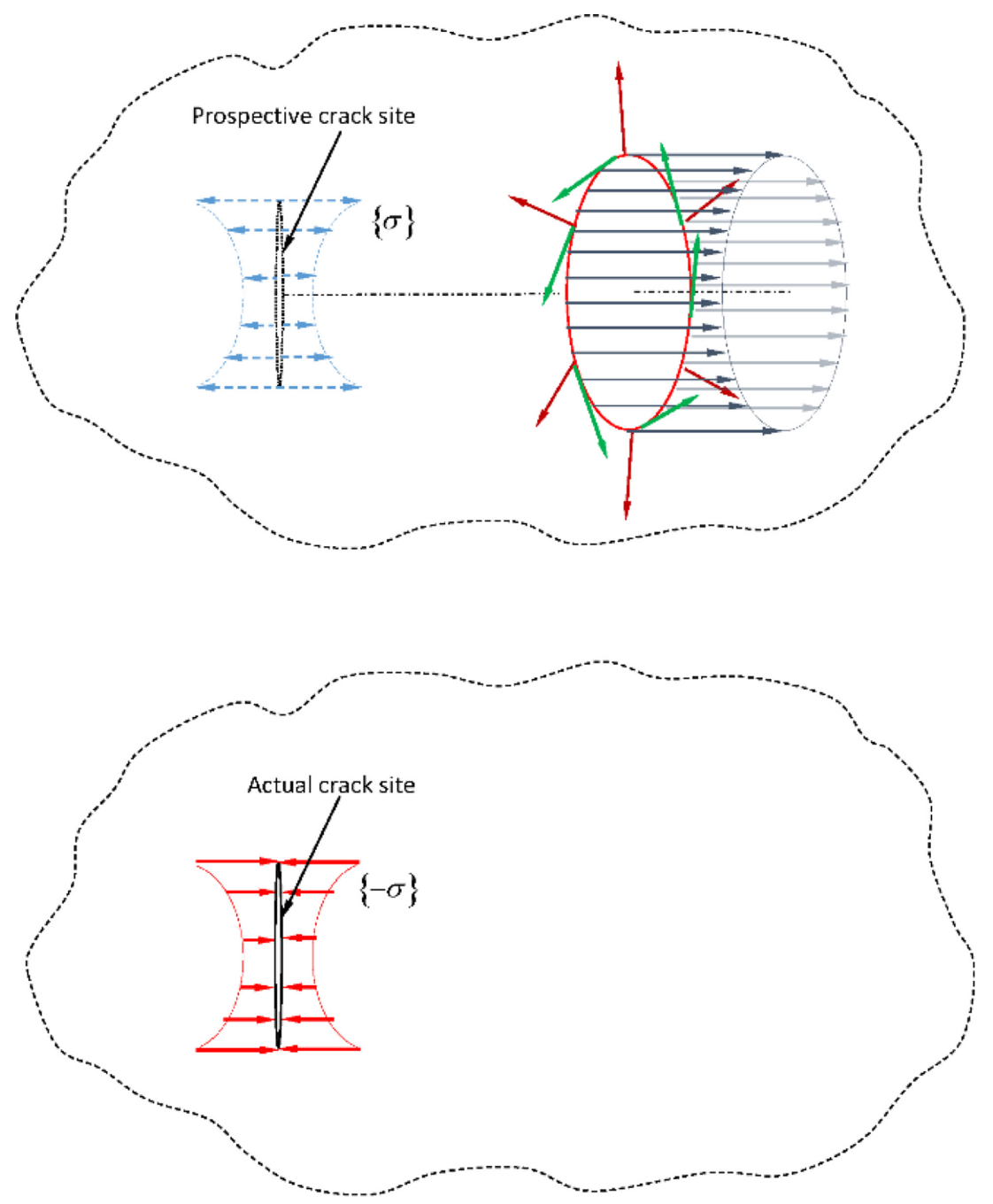

Figure 4: Superposition of two solutions for deriving the Green's function of a cracked medium under singular loading sources: (a) uncracked medium under singular ring-shaped source, (b) cracked medium under opposite tractions acting on crack surfaces.

displacements $u_{o}^{q_{r}}(r)$ on the plane $\mathrm{z}=0$ (Fig. 6) of the homogeneous medium are known [20]. Since the $\tau_{z y}$ on the plane $\mathrm{z}=0$ are zero due to the symmetry of the two loading sources, each sub-domain $\mathrm{z}>0$ or $\mathrm{z}<0$ (Fig. 6) can be considered as a half space. Having now the solution for the half space under singular ring-shaped source, the displacement distribution on the interface between the two materials (Fig. 5) can be obtained [16, 17]:

$$
u_{0}(r, 0)=-\frac{q_{\vartheta}}{G_{1}+G_{2}} \frac{1}{\pi r} f(r)
$$




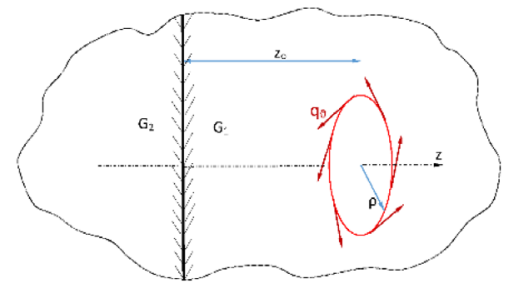

(a)
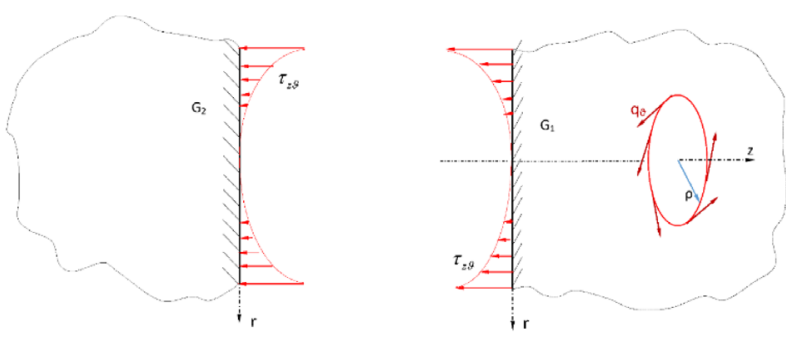

(b)

Figure 5: (a) A dissimilar elastic medium, (b) Decomposition of the dissimilar elastic medium to two half spaces.

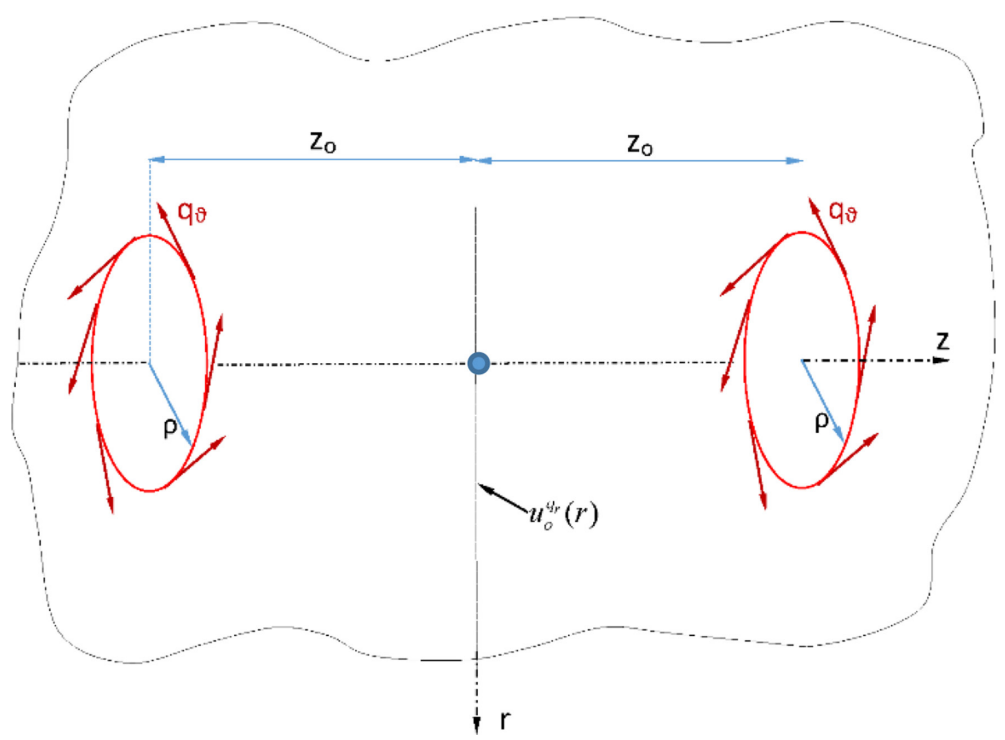

Figure 6: Homogeneous medium under two same loading sources.

$$
f(r)=\sqrt{(r+\rho)^{2}+z_{0}^{2}} E(\pi / 2, \kappa)-\frac{\left(r^{2}+\rho^{2}+z_{0}^{2}\right)}{\sqrt{(r+\rho)^{2}+z_{0}^{2}}} K(\pi / 2, \kappa
$$

$K(\pi / 2, \kappa), E(\pi / 2, \kappa)$ are the complete elliptic integrals with modulus 

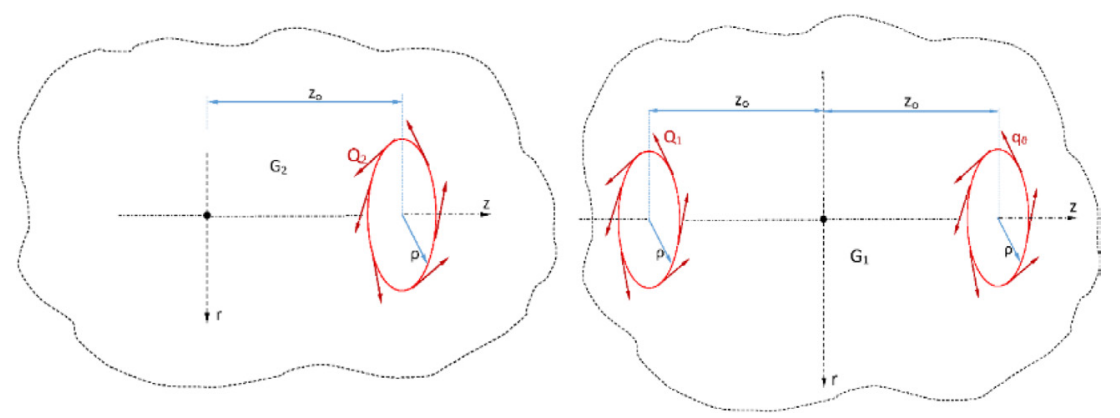

Figure 7: (a) Homogeneous elastic medium with shear modulus $G_{2}$ subjected to a fictitious load $\mathrm{Q}_{2}$ causing the displacement distribution $u_{0}(r, 0)$ given by eqn (1), (b) Homogeneous elastic medium with shear modulus $\mathrm{G}_{1}$ subjected to the actual loading source $\mathrm{q}_{\vartheta}$ and to the fictitious one $\mathrm{Q}_{1}$ causing the displacement distribution $u_{0}(r, 0)$ given by eqn (1).

$$
\kappa^{2}=\frac{4 r \rho}{(r+\rho)^{2}+z_{0}^{2}} \quad, \quad 0 \leq \kappa^{2} \leq 1
$$

Then, fictitious loads $\mathrm{Q}_{2}$ and $\mathrm{Q}_{1}$ can be applied on the homogeneous media with shear modulus $\mathrm{G}_{2}$ and $\mathrm{G}_{1}$ respectively (Fig. 7a and b) in order to cause the displacement distribution $u_{0}(r, 0)$ given by eqn (1).

Applying the existing solution for the displacements [20] on the plane $\mathrm{z}=0$ in the two homogeneous elastic mediums shown in Fig. 7a and b, and using eqn (1)

$$
\begin{gathered}
-\frac{Q_{2}}{2 G_{2}} \frac{1}{\pi r} f(r)=-\frac{q_{\vartheta}}{G_{1}+G_{2}} \frac{1}{\pi r} f(r) \\
-\frac{\left(q_{\vartheta}+Q_{1}\right)}{2 G_{1}} \frac{1}{\pi r} f(r)=-\frac{q_{\vartheta}}{G_{1}+G_{2}} \frac{1}{\pi r} f(r)
\end{gathered}
$$

the suitable values for the fictitious loads $\mathrm{Q}_{1}$ and $\mathrm{Q}_{2}$ causing the displacement distribution $u_{o}(r, 0)$ given by eqn (1) can be obtained:

$$
\begin{gathered}
Q_{2}=\frac{2 G_{2}}{G_{1}+G_{2}} q_{\vartheta} \\
Q_{1}=\frac{G_{1}-G_{2}}{G_{1}+G_{2}} q_{\vartheta}
\end{gathered}
$$

Using the above values for $\mathrm{Q}_{1}, \mathrm{Q}_{2}$, the displacement distribution on the plane $\mathrm{z}=0$ of the homogeneous media shown in Fig. 7a and $\mathrm{b}$ is the same as the displacement distribution on the interface $\mathrm{z}=0$ of the dissimilar medium shown in Fig. 5a. Then, the sub-domain $z \geq 0$ of the dissimilar medium shown in Fig. 5a has the same loading and boundary conditions (on $\mathrm{z}=0$ ) with the sub-domain $z \geq 0$ of the homogeneous medium shown in Fig. $7 \mathrm{~b}$. 
Therefore, the solution for $z \geq 0$ of the dissimilar medium shown in Fig. 5a is the same as the solution for $z \geq 0$ of the homogeneous medium shown in Fig. 7b:

$$
\begin{gathered}
u(r, z)=-\frac{q_{\vartheta}}{2 G_{1}} \frac{1}{\pi r} f_{1}(r, z)-\frac{Q_{1}}{2 G_{1}} \frac{1}{\pi r} f_{2}(r, z) \quad z \geq 0 \\
f_{1}(r, z)=\sqrt{(r+\rho)^{2}+\left(z-z_{o}\right)^{2}} E\left(\pi / 2, \kappa_{1}\right)-\frac{r^{2}+\rho^{2}+\left(z-z_{o}\right)^{2}}{\sqrt{(r+\rho)^{2}+\left(z-z_{o}\right)^{2}}} K\left(\pi / 2, \kappa_{1}\right) \\
f_{2}(r, z)=\sqrt{(r+\rho)^{2}+\left(z+z_{o}\right)^{2}} E\left(\pi / 2, \kappa_{2}\right)-\frac{r^{2}+\rho^{2}+\left(z+z_{o}\right)^{2}}{\sqrt{(r+\rho)^{2}+\left(z+z_{o}\right)^{2}}} K\left(\pi / 2, \kappa_{2}\right) \\
\kappa_{1}^{2}=\frac{4 r \rho}{(r+\rho)^{2}+\left(z-z_{o}\right)^{2}} \quad 0 \leq \kappa_{1}^{2} \leq 1 \\
\kappa_{2}^{2}=\frac{4 r \rho}{(r+\rho)^{2}+\left(z+z_{o}\right)^{2}} \quad 0 \leq \kappa_{2}^{2} \leq 1
\end{gathered}
$$

Taking into account the following eqns (13) and (14) obtained by [21, 22] respectively,

$$
\begin{gathered}
\frac{1}{\kappa}\left[\left(2-\kappa^{2}\right) K(\pi / 2, \kappa)-2 E(\pi / 2, \kappa)\right]=Q_{1 / 2}(\gamma) \\
\frac{1}{\pi \sqrt{r \rho}} Q_{1 / 2}(\gamma)=\int_{0}^{\infty} e^{-z t} J_{1}(r t) J_{1}(\rho t) d t \\
\gamma=\frac{r^{2}+\rho^{2}+z^{2}}{2 r \rho}
\end{gathered}
$$

the solution for the dissimilar medium shown in Fig. 5a can be written in terms of Bessel functions:

$$
\begin{gathered}
u_{\vartheta}(r, z)=\frac{1}{4 \pi G_{1}}\left\{\int_{0}^{\infty} g_{1}(r, z, \omega) d \omega+\frac{G_{1}-G_{2}}{G_{1}+G_{2}} \int_{0}^{\infty} g_{2}(r, z, \omega) d \omega\right\} \quad z \geq 0 \\
\tau_{z \vartheta}(r, z)=G_{1} \frac{\partial u_{\vartheta}(r, z)}{\partial z}=-\frac{1}{4 \pi}\left\{\int_{0}^{\infty} \omega g_{1}(r, z, \omega) d \omega+\frac{G_{1}-G_{2}}{G_{1}+G_{2}} \int_{0}^{\infty} \omega g_{2}(r, z, \omega) d \omega\right\} \quad z \geq 0 \\
u_{\vartheta}(r, z)=\frac{1}{2 \pi\left(G_{1}+G_{2}\right)} \int_{0}^{\infty} g_{1}(r, z, \omega) d \omega \quad z \leq 0 \\
\tau_{z \vartheta}(r, z)=\frac{G_{2}}{4 \pi\left(G_{1}+G_{2}\right)} \int_{0}^{\infty} \omega g_{2}(r, z, \omega) d \omega \quad z \leq 0
\end{gathered}
$$

where:

$$
\begin{aligned}
& g_{1}(r, z, \omega)=e^{-\left(z-z_{o}\right) \omega} J_{1}(r \omega) J_{1}(\rho \omega) \\
& g_{2}(r, z, \omega)=e^{-\left(z+z_{o}\right) \omega} J_{1}(r \omega) J_{1}(\rho \omega)
\end{aligned}
$$




\subsection{Solution for the interfacial crack of a dissimilar medium under crack surface tractions}

Let us now assume a dissimilar medium containing a penny-shaped crack. To this medium, only the crack surfaces are subjected to equal and opposite tractions. Since solution exists only for a homogeneous medium containing crack under equal and opposite tractions [15], the dissimilar medium should be substituted by two different homogeneous media, containing sub-domains with the same loading and boundary conditions with the sub-domains of the dissimilar medium.

Taking into account ref. [23] the displacements on the plane $z=0$, of the half space $G_{1}$ in Fig. 8 is given by the following equation:

$$
u_{1}(r, 0)=\frac{1}{4 \pi^{2} G_{1}} h(r)
$$

On the same plane $\mathrm{z}=0$ of half-space $\mathrm{G}_{2}$ (Fig. 8) the displacements are:

$$
\begin{gathered}
u_{2}(r, 0)=\frac{1}{4 \pi^{2} G_{2}}(-h(r)) \\
h(r)=\int_{0}^{a} \frac{-\tau_{z \theta}^{c}(\eta)}{\sqrt{\eta r}} Q_{1 / 2}(x) d \eta+\int_{a}^{\infty} \frac{\tau_{z \theta}^{1,2}(\eta)}{\sqrt{\eta r}} Q_{1 / 2}(x) d \eta \\
x=\frac{\eta^{2}+r^{2}}{2 \eta r}
\end{gathered}
$$

Assuming that the two half spaces are perfectly bonded, the condition

$$
u_{1}(r, 0)=u_{2}(r, 0)
$$

yields

$$
u_{1}(r, 0)=u_{2}(r, 0)=0
$$

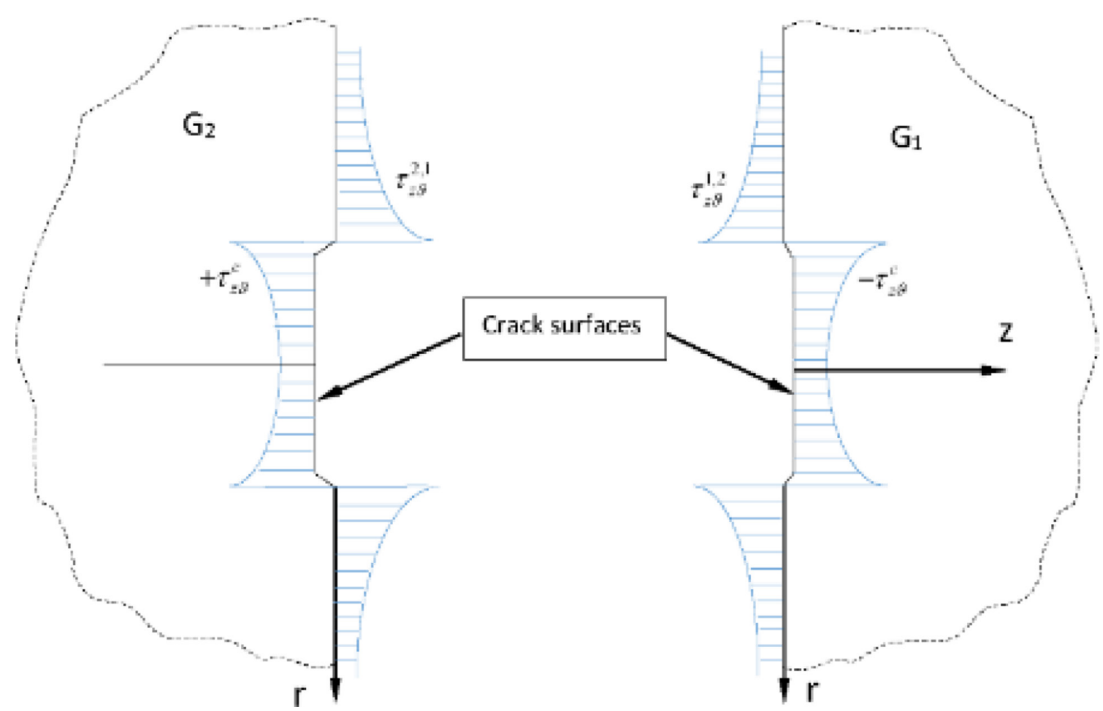

Figure 8: Decomposition of the cracked dissimilar medium to two half spaces. 
Therefore, the sub-domain $z \geq 0$ of the cracked dissimilar medium (Fig. 8) has the same loading and boundary conditions and the same material properties as the sub-domain $z \geq 0$ of the cracked homogeneous medium shown in Fig. 9a. Similarly, the sub-domain $z \leq 0$ of the dissimilar cracked medium has the same loading and boundary conditions and the same material properties as the sub-domain $z \leq 0$ of the cracked homogeneous medium shown in Fig. 9b. Since the solution for a homogeneous domain containing a penny-shaped crack subjected to opposite and equal surface tractions is known [15]

$$
u(r, z)=-\frac{1}{G_{i}} \int_{0}^{\infty} C(s) J_{1}(r s) e^{-s z} d s
$$
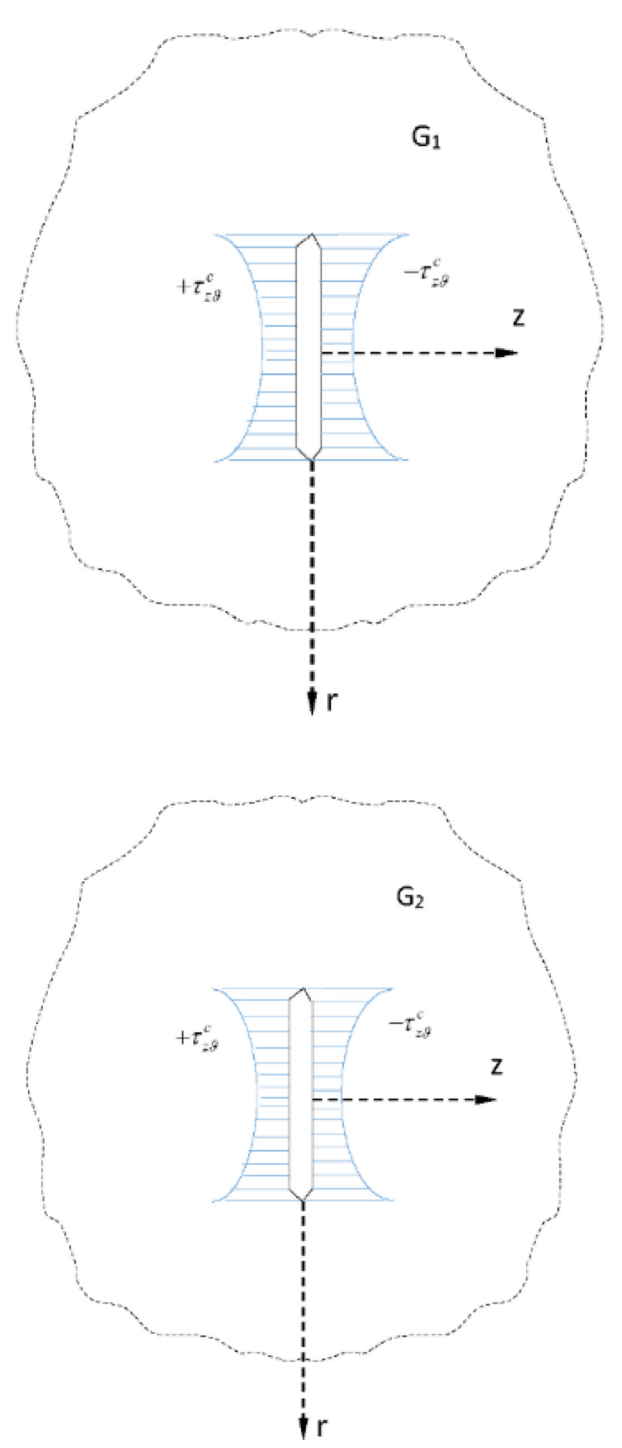

Figure 9: (a) Cracked homogeneous medium with shear modulus $G_{1}$, (b) Cracked homogeneous medium with shear modulus $G_{2}$. 


$$
C(s)=\sqrt{\frac{2 s}{\pi}} \int_{0}^{a} \frac{J_{3 / 2}(s t)}{\sqrt{t}} d t \int_{0}^{t} \frac{\xi^{2} \tau_{z \theta}^{c}(\xi)}{\sqrt{t^{2}-\xi^{2}}} d \xi
$$

the expression for $\tau_{z c}^{c}$ in the above equations can be substituted by the opposite stresses $\tau_{z \vartheta}$ given by eqns (17) and (19). Then, superposition of the solutions of the media shown in Fig. $4 \mathrm{a}$ and $\mathrm{b}$ (taking into account only the tangential component of the loading source) yield the required Green's function for the dissimilar domain containing interfacial penny-shaped crack, under tangential ring-shaped singular loading source (Fig. 2a). The calculation of the resulted quadruplicate integrals is the main challenge. Analytic solution simplifying the quadruplicate integrals to single ones has been achieved by the author [16]. This Green's function is summarized in the Appendix 1. Following the same concept, Green's function for the dissimilar medium containing external interfacial crack, subjected to ring-shaped tangential loading source, as well as Green's functions for homogeneous mediums containing penny-shaped crack under ring-shaped axial and radial loading sources [17-19] are summarized in Appendices 2 to 3 respectively.

\section{NUMERICAL RESULTS}

Graphical representation of the solutions provided in Appendices 1-3 can be obtained for some typical cases of dissimilar or homogeneous crack problems under singular ring-shaped sources.

Example 1: Dissimilar material containing an interfacial penny-shaped crack and a singular tangential ring-shaped loading source.

A dissimilar space composed by aluminum (on $\mathrm{z}>0$ ) and steel (on $\mathrm{z}<0$ ) with shear modulus $\mathrm{G}_{1}=26 \mathrm{GPa}$ and $\mathrm{G}_{2}=75 \mathrm{GPa}$, respectively, contains an interfacial (on $\mathrm{z}=0$ ) penny-shaped crack with radius $\alpha=0.01 \mathrm{~m}$. The above dissimilar space is subjected to a singular, ringshaped, tangential loading source with radius $\rho=0.08 \mathrm{~m}$ and magnitude $2 \pi \rho q_{\vartheta}=1$ located on the plane $\mathrm{z}_{\mathrm{o}}=0.08 \mathrm{~m}$. With the aid of the solution given in Appendix 1, the shear stress distribution $\tau_{z \vartheta}$ versus $\mathrm{r}$ for three planes $\mathrm{z}=0.010 \mathrm{~m}, 0.013 \mathrm{~m}$, and $0.016 \mathrm{~m}$ is demonstrated in Fig. 10. According to this figure, the peak values of the shear stress distribution take place

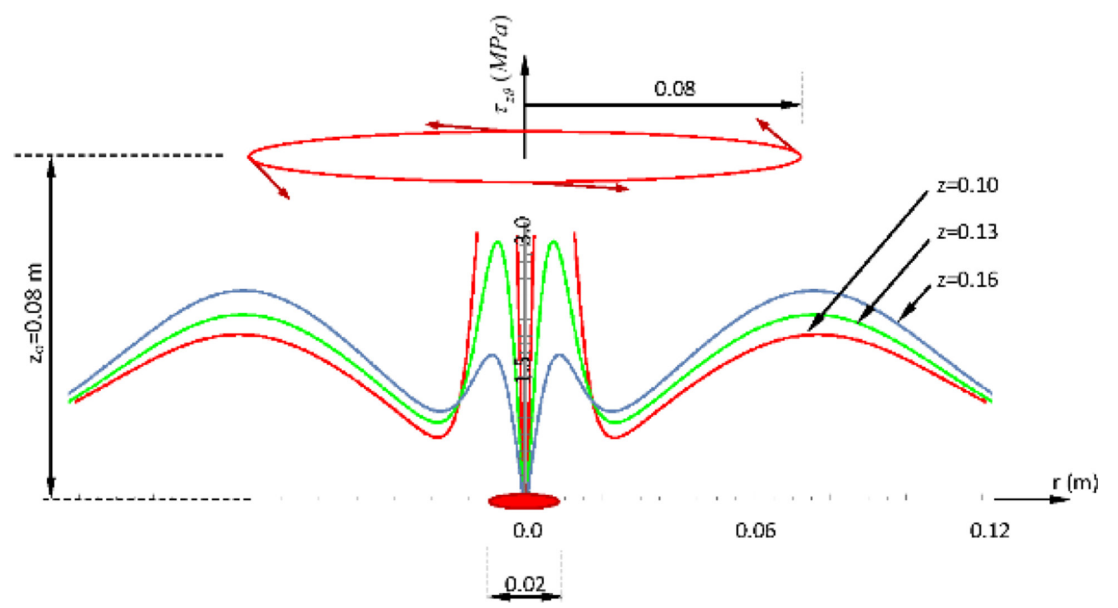

Figure 10: Shear stress $\tau_{z \vartheta}$ distribution on the sub-domain $\mathrm{z}>0$ (material $\mathrm{G}_{1}$ ) of a dissimilar medium containing a penny-shaped crack and ring-shaped source. 
on the crack tip $(\mathrm{r}=0.01 \mathrm{~m})$ and on the radius of the singular loading source $(\mathrm{r}=0.08 \mathrm{~m})$. As the distance of the reference plane moves from the plane of the crack toward the plane of the loading source, the peak value on $r=0.01 \mathrm{~m}$ decreases while the peak value on $r=0.08 \mathrm{~m}$ increases.

Example 2: Dissimilar material containing an external interfacial crack and a singular, Tangential, ring-shaped loading source, lying on the interface

A same aluminum/steel dissimilar space contains an external crack with internal radius $0.010 \mathrm{~m}$ and infinite external radius. The dissimilar space is subjected to a ring-shaped, tangential loading source $2 \pi \rho q_{\vartheta}=1$ with radius $\rho=0.03 \mathrm{~m}$ located on the interface $\mathrm{z}=0$. Using the solution given in Appendix 2, the shear stress distribution $\tau_{z 9}$ versus $r$ for three planes $\mathrm{z}=0.04 \mathrm{~m}, 0.06 \mathrm{~m}$, and $0.08 \mathrm{~m}$ is shown in Fig. 11. It can be observed that the peak values take place on $r=0.03 \mathrm{~m}$ and $\mathrm{r}=0.10 \mathrm{~m}$, that is, on the vicinity of the singular source and the crack tip, respectively. As the distance $\mathrm{z}$ from the above singularities increases, the values of the shear stress decreases.

Example 3: Homogeneous material containing a penny-shaped crack and a singular, ringshaped axial and radial loading source

A homogeneous space made by steel contains a penny-shaped crack with radius $\alpha=0.01 \mathrm{~m}$. The domain is subjected to a singular, ring-shaped loading source with axial and radial components $2 \pi \rho q_{z}=1$ and $2 \pi \rho q_{r}=1$ respectively, and radius $\rho=0.08 \mathrm{~m}$. The distance of the plane of the loading source from the plane of the crack surface is $z_{0}=0.08 \mathrm{~m}$. With the aid of the solution obtained by Appendix 3, the axial displacement $u_{z z}$ versus $\mathrm{r}$ is demonstrated in Fig. 12 for the planes $\mathrm{z}=0.01 \mathrm{~m}$ (near to the crack surface) and $\mathrm{z}=0.07 \mathrm{~m}$ (near to the loading source). In the above figure, peak values for the displacement $u_{z z}$ can be observed on $\mathrm{r}=0.01 \mathrm{~m}$ (crack tip) and $\mathrm{r}=0.08 \mathrm{~m}$ (perimeter of the loading source). As the reference plane moves from $\mathrm{z}=0.01 \mathrm{~m}$ to $\mathrm{z}=0.07 \mathrm{~m}$, a rapid decrease of the peak value of $u_{z z}$ on $\mathrm{r}=0.01 \mathrm{~m}$ and a considerable increase of its value on $\mathrm{r}=0.08 \mathrm{~m}$ happen.

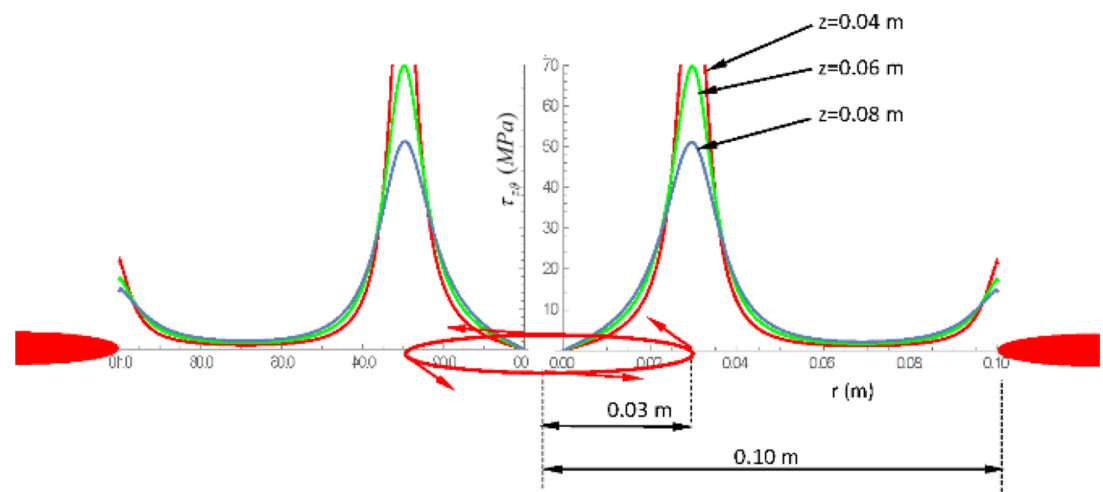

Figure 11: Shear stress $\tau_{z \vartheta}$ distribution on the sub-domain $\mathrm{z}>0$ (material $\mathrm{G}_{1}$ ) of a dissimilar medium containing an external annular crack and a ring-shaped source. 


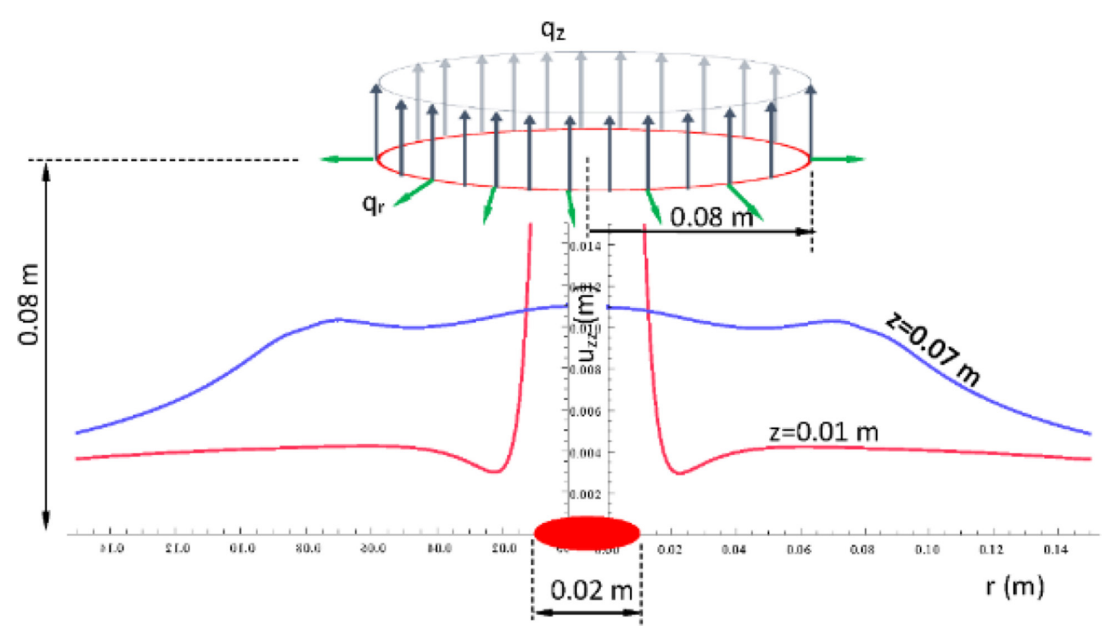

Figure 12: Axial displacement distribution $u_{z z}$ on the sub-domain $\mathrm{z}>0$ of a homogeneous material containing a penny-shaped crack and a singular, ring-shaped loading source with axial and radial components.

\section{CONCLUSIONS}

A review of Green's functions developed by the author for dissimilar or homogeneous media containing axi-symmetric cracks and singular, ring-shaped loading sources has been summarized.

A brief presentation of the methodology for developing the above Green's functions has been presented.

Numerical results for three examples have been demonstrated for three cases: (1) Dissimilar material containing a penny-shaped interfacial crack and a singular tangential, ring-shaped loading source, (2) Dissimilar material containing an external interfacial crack and a singular tangential, ring-shaped loading source located on the interface, and (3) Homogeneous material containing a penny-shaped crack and an axial/radial singular loading source.

In all examples, the field values take peak values in the vicinity of the crack tip and the singular source.

The above Green's functions can be used for BE modeling of finite axi-symmetric cracked solids under axial and torsional loads (e.g., cracked shafts). The advantages of using the above Green's functions are: No multi-domain modeling and no discretization of the crack surfaces is necessary.

\section{REFERENCES}

[1] Xiaoping, L. \& Wei-Liang, W., A new subregion boundary element technique based on the domain decomposition method. Engineering Analysis with Boundary Elements, 29, pp. 944-952, 2005.

http://dx.doi.org/10.1016/j.enganabound.2005.08.001

[2] Gao, X-W., Guo, L. \& Zhang, C., Three-step multi-domain BEM solver for nonhomogeneous material problems. Engineering Analysis with Boundary Elements, 31, pp. 965-973, 2007.

http://dx.doi.org/10.1016/j.enganabound.2007.06.002 
[3] Ribeiro, D.B. \& Paiva, J.B., An alternative multi-region BEM technique for threedimensional elastic problems. Engineering Analysis with Boundary Elements, 33, pp. 499-507, 2009.

http://dx.doi.org/10.1016/j.enganabound.2008.08.016

[4] Ramšak, M. \& Škerget, L., 3D multidomain BEM for solving the Laplace equation. Engineering Analysis with Boundary Elements, 31, pp. 528-538, 2007.

http://dx.doi.org/10.1016/j.enganabound.2006.10.006

[5] Ramšak, M. \& Škerget, L., 3D multidomain BEM for a poisson equation. Engineering Analysis with Boundary Elements, 33, pp. 689-694, 2009.

http://dx.doi.org/10.1016/j.enganabound.2008.09.009

[6] Gao, X-W. \& Wang, J., Interface integral BEM for solving multi-medium heat conduction problems. Engineering Analysis with Boundary Elements, 33, pp. 539-546, 2009. http://dx.doi.org/10.1016/j.enganabound.2008.08.009

[7] Ramšak, M. \& Škerget, L., A multidomain boundary element method for two equation turbulence models. Engineering Analysis with Boundary Elements, 29, pp. 1086-1103, 2005. http://dx.doi.org/10.1016/j.enganabound.2005.07.007

[8] Davi, G. \& Milazzo, A., Multidomain boundary integral equation for piezoelectric materials fracture mechanics. International Journal of Solids and Structures, $\mathbf{3 8}$, pp. 7065-7078, 2001. http://dx.doi.org/10.1016/S0020-7683(00)00416-9

[9] Galvis, A.F. \& Sollero, P., Boundary element analysis of crack problems in polycrystalline materials. Procedia Materials Science, 3, pp. 1928-1933, 2014. http://dx.doi.org/10.1016/j.mspro.2014.06.311

[10] Phan, A.-V. \& Mukherjee, S., The multi-domain boundary contour method for interface and dissimilar material problems. Engineering Analysis with Boundary Elements, 33, pp. 668-677, 2009. http://dx.doi.org/10.1016/j.enganabound.2008.10.004

[11] Zhao, Y.F., Zhao, M.H. \& Pan, E., Displacement discontinuity analysis of a nonlinear interfacial crack in three-dimensional transversely isotropic magneto-electro-elastic bi-materials. Engineering Analysis with Boundary Elements, 61, pp. 254-264, 2015. http://dx.doi.org/10.1016/j.enganabound.2015.08.001

[12] Lei, J., Garcia-Sanchez, F., Wünsche, M., Zhang, C., Wang, Y.-S. \& Saez, A., Dynamic analysis of interfacial crack problems in anisotropic bi-materials by a time-domain BEM. Engineering Analysis with Boundary Elements, 76, pp. 1996-2010, 2009. http://dx.doi.org/10.1016/j.engfracmech.2009.05.006

[13] Lei, J., Garcia-Sanchez, F. \& Zhang, C., Determination of dynamic intensity factors and time-domain BEM for interfacial cracks in anisotropic piezoelectric materials. International Journal of Solids and Structures, 50, pp. 1482-1493, 2013. http://dx.doi.org/10.1016/j.ijsolstr.2013.01.018

[14] Yang, B., Pan, E. \& Tewary, V.K., Three-dimensional Green's functions of steady-state motion in anisotropic half-spaces and bimaterials. Engineering Analysis with Boundary Elements, 28, pp. 1069-1082, 2004.

http://dx.doi.org/10.1016/j.enganabound.2004.03.004

[15] Kassir, M.K. \& Sih, G.C., Three Dimensional Crack Problems, Noordhoff: Leyden, 1975.

[16] Pavlou, D.G., Boundary-integral equation analysis of twisted internally cracked axisymmetric biomaterial elastic solids. Computational Mechanics, 29, pp. 254-264, 2002. http://dx.doi.org/10.1007/s00466-002-0338-7 
[17] Pavlou, D.G., Green's functions for the biomaterial elastic solid containing interface crack. Engineering Analysis with Boundary Elements, 26, pp. 845-853, 2002. http://dx.doi.org/10.1016/S0955-7997(02)00052-8

[18] Mavrothanasis, F.I. \& Pavlou, D.G., Mode-I stress intensity factor derivation by a suitable Green's function. Engineering Analysis with Boundary Elements, 31, pp. 184-190, 2007. http://dx.doi.org/10.1016/j.enganabound.2006.08.004

[19] Mavrothanasis, F.I. \& Pavlou, D.G., Green's function for KI determination of axisymmetric elastic solids containing external circular crack. Engineering Fracture Mechanics, 75, pp. 1891-1905, 2008.

http://dx.doi.org/10.1016/j.engfracmech.2007.08.014

[20] Kermanidis, T., A numerical solution for axially symmetric elasticity problems. International Journal of Solids and Structures, 11, pp. 493-500, 1975.

http://dx.doi.org/10.1016/0020-7683(75)90084-0

[21] Bakr, A.A. \& Fenner, R.T., Use of the Hankel transform in boundary integral methods for axisymmetric problems. International Journal of Numerical Methods in Engineering, 19, pp. 1765-1769, 1983.

http://dx.doi.org/10.1002/nme.1620191204

[22] Gradshteyn, I.S. \& Ryzhik, I.M., Table of Integrals, Series, and Products, Academic Press: New York, 2000.

[23] Hasegawa, H., Green's functions for axisymmetric surface force problems of an elastic halfspace and their application. Theoretical and Applied Mechanics, 32, pp. 291-300, 1984.

Appendix 1: Green's function for dissimilar elastic space containing an interfacial penny-shaped crack and a tangential, ring-shaped loading source.

Region $\mathrm{z}>0$

$$
\begin{gathered}
u_{\vartheta}(r, z)=\frac{1}{4 \pi G_{1}}\left\{\int_{0}^{\infty} e^{-\left(z-z_{0}\right) \omega} J_{1}(r \omega) J_{1}(\rho \omega) d \omega+\frac{G_{1}-G_{2}}{G_{1}+G_{2}} \int_{0}^{\infty} e^{-\left(z+z_{0}\right) \omega} J_{1}(r \omega) J_{1}(\rho \omega) d \omega\right\} \\
-\frac{1}{2 \pi^{2} G_{1}}\left\{\begin{array}{l}
a \\
j \\
0
\end{array} B_{0}(t) B_{21}(t) d t+\frac{G_{1}-G_{2}}{G_{1}+G_{2}} \int_{0}^{a} B_{0}(t) B_{22}(t) d t\right\}
\end{gathered}
$$

Region $\mathbf{z}<0$

$$
u_{\vartheta}^{K_{i}^{1}}(r, z)=\frac{1}{2 \pi\left(\mu_{1}+\mu_{2}\right)}\left\{\int_{0}^{\infty} e^{-\left(z-z_{0}\right) \omega} J_{1}(r \omega) J_{1}(\rho \omega) d \omega\right\}-\frac{1}{2 \pi^{2}\left(\mu_{1}+\mu_{2}\right)} \int_{0}^{a} B_{0}(t) B_{21}(t) d t
$$

The functions $B_{o}(t), B_{21}(t), B_{22}(t)$ can be obtained from Pavlou [16].

Appendix 2: Green's function for dissimilar elastic space containing an interfacial external crack and a tangential, ring-shaped loading source.

Region $\mathrm{z}>0$

$$
\begin{gathered}
u_{\vartheta}(r, z)=\frac{1}{4 \pi G_{1}}\left\{\int_{0}^{\infty} e^{-\left(z-z_{0}\right) \omega} J_{1}(r \omega) J_{1}(\rho \omega) d \omega+\frac{G_{1}-G_{2}}{G_{1}+G_{2}} \int_{0}^{\infty} e^{-\left(z+z_{0}\right) \omega} J_{1}(r \omega) J_{1}(\rho \omega) d \omega\right\} \\
-\frac{1}{2 \pi^{2} G_{1}}\left\{\int_{a}^{\infty} A_{0}(t) A_{21}(t) d t+\frac{G_{1}-G_{2}}{G_{1}+G_{2}} \int_{a}^{\infty} A_{0}(t) A_{22}(t) d t\right\}
\end{gathered}
$$


Region $\mathbf{z}<0$

$$
u_{\vartheta}(r, z)=\frac{1}{2 \pi\left(G_{2}+G_{1}\right)}\left\{\int_{0}^{\infty} e^{-\left(z-z_{0}\right) \omega} J_{1}(r \omega) J_{1}(\rho \omega) d \omega\right\}-\frac{1}{2 \pi^{2}\left(G_{2}+G_{1}\right)} \int_{a}^{\infty} A_{0}(t) A_{21}(t) d t
$$

The functions $A_{o}(t), A_{21}(t), A_{22}(t)$ can be obtained from Pavlou [17].

Appendix 3: Green's function for homogeneous elastic space containing a penny-shaped crack and an axial/radial, ring-shaped loading source.

\section{(a) Radial loading source}

$$
u_{r r}(r, z)=u_{r r}^{o}(r, z)+u_{r r}^{c}(r, z), u_{z r}(r, z)=u_{z r}^{o}(r, z)+u_{z r}^{c}(r, z)
$$

where:

$$
\begin{gathered}
u_{r r}^{o}=\frac{1}{16 \pi(1-v) G}\left[(3-4 v) \int_{0}^{\infty} e^{-\left(z-z_{o}\right) s} J_{1}(r s) J_{1}(\rho s) d s-\left(z-z_{o}\right) \int_{0}^{\infty} s e^{-\left(z-z_{o}\right) s} J_{1}(r s) J_{1}(\rho s) d s\right] \\
u_{r r}^{c}=\frac{1}{16 \pi^{2}(1-v) G}\left[\frac{z}{1-2 v} \int_{0}^{a} A_{2}^{I N}(t) L_{R A D}(t) d t-\int_{0}^{a} A_{1}^{I N}(t) L_{R A D}(t) d t\right] \\
u_{z r}^{o}=\frac{1}{16 \pi(1-v) G r}\left[\frac{1}{2} \int_{0}^{\infty} \int_{0}^{-\left(z-z_{o}\right) s} J_{1}(r s) J_{1}(\rho s) d s+\frac{\left(z-z_{o}\right)^{2}+\rho^{2}-r^{2}}{2\left(z-z_{o}\right)}\right] \\
u_{z r}^{c}=\frac{1}{16 \pi^{2}(1-v) G}\left[\frac{z}{1-v} \int_{0}^{a} B_{2}^{I N}(t) L_{R A D}(t) d t-2 \int_{0}^{a} B_{1}^{I N}(t) L_{R A D}(t) d t\right]
\end{gathered}
$$

(b) Axial loading source

$$
u_{z z}(r, z)=u_{z z}^{o}(r, z)+u_{z z}^{c}(r, z), u_{z r}(r, z)=u_{z r}^{o}(r, z)+u_{z r}^{c}(r, z)
$$

where:

$$
\begin{aligned}
& u_{z z}^{o}=\frac{1}{16 \pi(1-v) G}\left[(3-4 v) \int_{0}^{\infty} \int_{0}^{\infty} s e^{-\left(z-z_{o}\right) s} J_{0}(r s) J_{0}(\rho s) d s\right. \\
& u_{z z}^{c}=-\frac{v}{16 \pi^{2}(1-2 v) G}\left[\frac{z}{1-v} \int_{0}^{a} B_{2}^{I N}(t) L_{A X-I N}(t) d t+2 \int_{0}^{a} B_{1}^{I N}(t) L_{A X-I N}(t) d t\right]
\end{aligned}
$$




$$
\begin{aligned}
& u_{r z}^{o}=\frac{-\left(z-z_{o}\right)}{16 \pi(1-v) G r}\left[\frac{1}{2} \int_{0}^{\infty} \int_{0}^{-\left(z-z_{o}\right) s} J_{0}(r s) J_{0}(\rho s) d s-\frac{\left(z-z_{o}\right)^{2}+\rho^{2}-r^{2}}{2\left(z-z_{o}\right)}\right] \\
& \int_{r z}^{c}=-\frac{v}{16 \pi^{2}(1-v) G}\left[\frac{z}{1-2 v} \int_{0}^{a} A_{2}^{I N}(t) L_{A X-I N}(t) d t-\int_{0}^{a} A_{1}^{I N}(t) L_{A X-I N}(t) d t\right]
\end{aligned}
$$

The functions $A_{1}^{I N}(t), A_{2}^{I N}(t), B_{1}^{I N}(t), B_{2}^{I N}(t), L_{R A D}(t), L_{R A D}(t), L_{A X-I N}(t)$ can be obtained from Pavlou [17]. 